\title{
Sol-gel transition behavior near critical concentration and connectivity
}

\author{
Takamasa Sakai $^{1,2}$, Takuya Katashima ${ }^{3}$, Takuto Matsushita ${ }^{1}$ and Ung-il Chung ${ }^{1}$
}

A polymer cross-linking system undergoes a phase transition from liquid to solid at a critical point, which is called the sol-gel transition. The sol-gel transition is often understood in the context of the lattice-based percolation model. Two parameters govern the sol-gel transition including the connectivity and polymer concentration. In this study, we independently tuned these parameters and experimentally accessed the sol-gel transition point as a function of connectivity and concentration using a model gel system (tetra-polyethylene glycol gel). The connectivity required to percolate the system continuously increased as the polymer concentration decreased, which is completely different from that predicted by the site-bond percolation model. The viscoelastic behavior at the critical points indicates that the fractal dimension of the percolation clusters deviated from the prediction of the lattice-based percolation model as the polymer concentration decreased. These results indicate that the lattice assumption cannot be applied for a gelling system prepared far below the overlapping concentration. Polymer Journal (2016) 48, 629-634; doi:10.1038/pj.2015.124; published online 20 January 2016

\section{INTRODUCTION}

When we cross-link a polymeric liquid, a sol-gel transition occurs, and an infinite three-dimensional (3D) polymer network is formed. In the vicinity of the critical point, various physical quantities exhibit unique power law behaviors. Many earlier studies have discussed the power law behaviors of the cluster growth, ${ }^{1-7}$ elastic modulus, ${ }^{8-10}$ viscosity ${ }^{11,12}$ and viscoelastic properties ${ }^{13-17}$ with regards to percolation. ${ }^{18,19}$

The percolation models approximate the space dimension as lattices and predict the network formation process. The two main classes of percolation models include the bond-percolation model and the site-percolation model. The bond-percolation model treats the situation in which all the lattice sites are occupied and the bonds between neighboring sites are formed at random. The bondpercolation model is often used to predict the network formation process from a molten and concentrated polymer system. ${ }^{1-9,11-17}$ However, the applicability of the bond-percolation model to a dilute system is limited because a massive amount of diluent exists in the system, and the sites (monomer units) do not fill the space. The site-percolation model involves sites that are partly occupied, and the bonds between the occupied sites are 'certainly' formed. This model seems to be a candidate for predicting the sol-gel transition in dilute systems. However, this situation cannot be reached experimentally because the connectivity between neighboring sites is not perfect in reality. To predict the sol-gel transition of a dilute system, we need to consider the effects of both the concentration and connectivity. This situation can be modeled using the site-bond percolation model. ${ }^{20}$ In this model, we can tune both the site occupation and bond formation.
However, to the best of our knowledge, application of the site-bond percolation model to a dilute system has not been examined experimentally, which may be because of the difficulty with independent control of the polymer connectivity and polymer concentration in the real system. To validate the applicability of the site-bond percolation, we need to develop a new system that enables us to tune the connectivity and concentration independently.

Recently, we successfully designed and fabricated a new class of model networks (that is, tetra-polyethylene glycol (tetra-PEG) gel). ${ }^{21-25}$ Tetra-PEG gels are formed by AB-type cross-link-coupling of two types of tetra-arm PEGs with amino groups and activated ester groups. The elementary reaction of the gelation reaction obeyed the second order reaction of amine and activated ester, suggesting that the prepolymers are premixed homogeneously and react efficiently. ${ }^{26,27}$ We directly estimated the connectivity based on the reaction conversion using infrared spectroscopy to determine the ratio of the reacted ends to all the reactive ends. The reaction conversion can be precisely tuned and was as high as $95 \% .^{28,29}$ Characterization using small-angle neutron scattering measurements indicated that almost no global heterogeneity existed in the regions up to $200 \mathrm{~nm}$ in size. ${ }^{30}$ The analyses of the small deformation and the fracture test indicated that the elasticity of the tetra-PEG gels simply originated from the chemical cross-links, and the contribution from entanglement couplings between network strands was negligible. ${ }^{29}$ Based on these results, the tetra-PEG gel is a promising model system with high homogeneity and controllability.

In this study, we investigated the sol-gel transition point as a function of connectivity and concentration. Here, we discuss the

\footnotetext{
${ }^{1}$ Department of Bioengineering, Graduate School of Engineering, The University of Tokyo, Tokyo, Japan; ${ }^{2}$ JST, PREST, Saitama, Japan and ${ }^{3}$ Department of Macromolecular Science, Graduate School of Science, Osaka University, Osaka, Japan

Correspondence: Dr T Sakai, Department of Bioengineering, Graduate School of Engineering, The University of Tokyo, 7-3-1 Hongo, Bunkyo-ku, Tokyo 113-8656, Japan. E-mail: cigamder@me.com

Received 26 August 2015; revised 19 October 2015; accepted 13 November 2015; published online 20 January 2016
} 


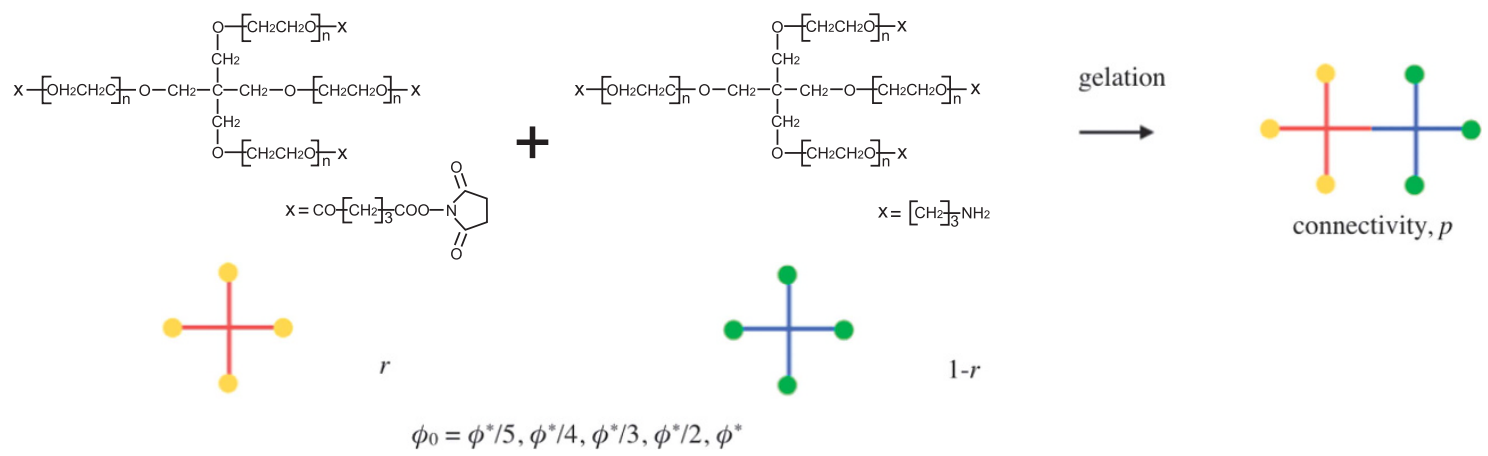

Figure 1 Schematic representation of the experimental design.

applicability of the site-bond percolation model to the sol-gel transition of a dilute system using the tetra-PEG gel as a model system (Figure 1). We constructed a sol-gel phase diagram tuning the prepolymer volume fraction of the tetra-arm prepolymers $\left(\phi_{0}\right)$, ranging from $\phi^{\star} / 6$ to $\phi^{*}$, where $\phi^{*}$ is the overlapping polymer volume fraction of the prepolymer. To tune the connectivity, we mixed tetra-PEG prepolymers with an unbalanced stoichiometry at each polymer volume fraction. In the vicinity of the critical points, we observed the power law behavior of the viscoelasticity. In addition, we compared the experimental results with the predictions from the percolation models and assessed their applicability to the sol-gel transition. These experimental results will contribute to the fundamental understanding of the sol-gel transition phenomena.

\section{EXPERIMENTAL PROCEDURE}

\section{Fabrication of the critical solution}

Tetra-amine-terminated PEG (tetra-PEG- $\mathrm{NH}_{2}$ ) and tetra-OSu-terminated PEG (tetra-PEG-OSu) were purchased from NOF Co. (Tokyo, Japan). Here, -OSu stands for $N$-hydroxysuccinimide. The molecular weight $\left(M_{\mathrm{W}}\right)$ of tetra-PEG$\mathrm{NH}_{2}$ and tetra-PEG-OSu was $20 \mathrm{~kg} \mathrm{~mol}^{-1}$. We combined stoichiometrically unbalanced amounts of tetra-PEG-NH $\mathrm{N}_{2}$ and tetra-PEG-OSu to control the reaction conversion in the final state. We prepared the samples using various initial polymer volume fractions $\left(\phi_{0}=0.034,0.017,0.011,0.0087,0.0070\right.$ and 0.0061 ). To determine the critical gelation molar ratios, the tetra-PEG-OSu to total tetra-PEG molar ratios $(r)$ were varied at each concentration, as shown in Table 1. Tetra-PEG-NH $\mathrm{N}_{2}$ and tetra-PEG-OSu were dissolved in a phosphate $\mathrm{D}_{2} \mathrm{O}$ buffer ( $\mathrm{pH}$ 7.4) and a phosphate-citric acid $\mathrm{D}_{2} \mathrm{O}$ buffer ( $\mathrm{pH} 5.8$ ), respectively. The ionic strengths of the buffer solutions were tuned from $5-35 \mathrm{~mm}$ in proportion to the concentration of the amine group. The two solutions were mixed and maintained for at least $12 \mathrm{~h}$ to achieve reaction completion.

\section{Viscoelastic measurement \\ The solution with tetra-PEG- $\mathrm{NH}_{2}$ and tetra-PEG-OSu was poured into the interstice of the double cylinder of a rheometer (MCR302; Anton Paar, Graz, Austria). The storage modulus $\left(G^{\prime}\right)$ and loss modulus $\left(G^{\prime \prime}\right)$ were measured $12 \mathrm{~h}$ after mixing. The angular frequency $(\omega)$ dependence of $G^{\prime}$ and $G^{\prime \prime}$ was measured with a strain amplitude $(\gamma)$ of $10-200 \%$ at $25^{\circ} \mathrm{C}$. The value of $\gamma$ was determined so that the measurement could be performed in the linear viscoelastic region.}

\section{Nuclear magnetic resonance (NMR) measurement}

The ${ }^{1} \mathrm{H}$ NMR spectra were measured using a JEOL Alpha series $(400 \mathrm{MHz})$ spectrometer. ${ }^{1} \mathrm{H}$ NMR $\left(\delta_{\text {ppm }}, \mathrm{D}_{2} \mathrm{O}\right): 1.75\left(\mathrm{q}, \mathrm{CH}_{2} \mathrm{CH}_{2} \mathrm{CH}_{2} \mathrm{NH}_{2}, J=6.50\right), 1.58$ (q, $\left.\mathrm{CH}_{2} \mathrm{CH}_{2} \mathrm{CH}_{2} \mathrm{NHCO}, J=6.65\right), 1.41\left(\mathrm{~m}, \mathrm{CH}_{2} \mathrm{CH}_{2} \mathrm{CH}_{2} \mathrm{CH}_{2} \mathrm{CH}_{2}\right)$ and 1.14 (m, $\mathrm{CH}_{2} \mathrm{CH}_{2} \mathrm{CH}_{2} \mathrm{CH}_{2} \mathrm{CH}_{2}$ ).
Table 1 The polymer volume fraction of tetra-PEG prepolymers $\left(\phi_{0}\right)$ and the range of the molar ratio of tetra-PEG-OSu $(r)$ of the critical solutions

\begin{tabular}{lc}
\hline$\phi_{0}[-]$ & \multicolumn{1}{c}{$r[-]$} \\
\hline 0.034 & $0.11,0.13,0.15,0.17,0.19,0.21$ \\
0.017 & $0.17,0.19,0.21,0.23,0.25,0.27$ \\
0.011 & $0.22,0.24,0.26,0.28,0.30,0.32$ \\
0.0087 & $0.27,0.29,0.31,0.33,0.35,037$ \\
0.0070 & $0.32,0.34,0.36,0.38,0.40,0.42$ \\
0.0061 & $0.40,0.42,0.44,0.46,0.48,0.50$
\end{tabular}

Abbreviations: tetra-PEG, tetra-polyethylene glycol; tetra-PEG-OSu, tetra-OSu-terminated PEG.

\section{RESULTS AND DISCUSSION}

Change in viscoelastic properties in the vicinity of the sol-gel transition point

Figure 2 shows the $\omega$ dependence of $G^{\prime}$ and $G^{\prime \prime}$ for mixtures of tetra-PEG prepolymers with various stoichiometric ratios $(r)$ at $\phi_{0}=0.034$. When $r<r_{\mathrm{c}}\left(r_{\mathrm{c}}=0.18\right), G^{\prime \prime}$ was $>G^{\prime}$ in the studied range, and the $\omega$ dependence of $G^{\prime \prime}$ exhibited the following relationship: $G^{\prime \prime} \sim \omega^{1}$. This tendency is similar to the typical terminal relaxation of polymer solutions. ${ }^{31}$ When $r>r_{\mathrm{c}}, G^{\prime}$ was $>G^{\prime \prime}$, and a plateau region for $G^{\prime}$ was observed at a low frequency, indicating that the system is percolated and in the gel state. At $r=r_{\mathrm{c}}$, the curves of $G^{\prime}$ and $G^{\prime \prime}$ overlapped with each other, and a power law behavior was observed, indicating the criterion of the sol-gel transition reported by Winter and Chambon. ${ }^{17}$ According to this criterion, we also determined the critical gelation molar ratios $\left(r_{\mathrm{c}}\right)$ at other polymer volume fractions $\left(\phi_{0}=0.017,0.011,0.0087,0.0070\right.$ and 0.0061$)$. Figure 3 shows the phase diagram of this system. The critical gelation molar ratio $\left(r_{\mathrm{c}}\right)$ increased with a decrease in $\phi_{0}$ and approached stoichiometry $(r=0.50)$, which suggests that a higher reaction conversion is required to percolate the system in the dilute solution region.

\section{Conversion of $r_{\mathrm{c}}$ to $p^{*}$}

To discuss the exact reaction conversion at the critical gelation point $\left(p^{\star}\right)$, we need to convert $r_{c}$ to $p^{*}$. We performed ${ }^{1} \mathrm{H}$ NMR measurements to directly estimate the amounts of reacted amide and unreacted amino groups. The signals from the $\beta$ carbon of the unreacted amino groups and the $\beta$ carbon of the amide bonds were observed at 1.75 p.p.m. (peak intensity $=a$ ) and 1.58 p.p.m. (peak intensity $=b$ ), respectively. The $a+b$ sum corresponds to the number of feed amino groups. Because the molar ratio of the amino groups to the activated ester groups was $(1-r) / r$, the total number of feed reactive groups including amino and activated ester groups 


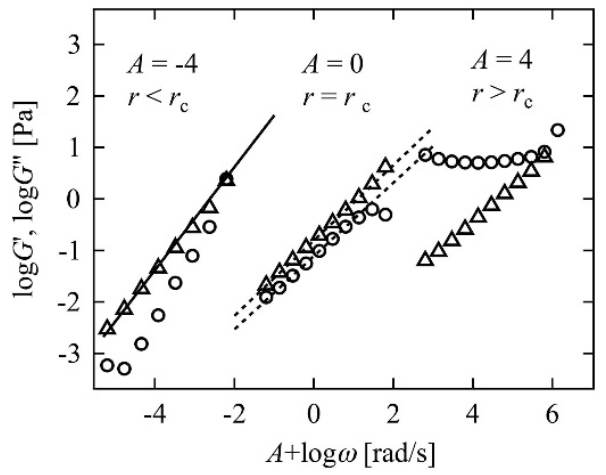

Figure 2 The value of $G^{\prime}$ and $G^{\prime \prime}$ as a function of $\omega$ in $\phi_{0}=0.034$ (circle; $G^{\prime}$, triangle; $\left.G^{\prime \prime}\right)$. The solid line shows the following relationship: $G^{\prime \prime} \sim \omega^{1}$. The dashed lines are the fitted curves showing the following relationship: $G^{\prime} \approx G^{\prime \prime} \sim \omega^{0.69}$. The curves were shifted sideways (factor $A$ ) to avoid overlap.

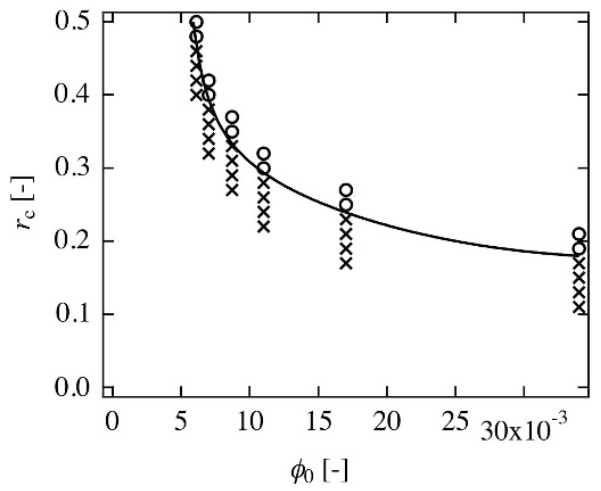

Figure 3 Phase diagram of off-stoichiometric tetra-polyethylene glycol (tetraPEG) system (circle, gel; cross, sol). The solid line shows the boundary line between the sol and gel phases.

corresponds to $(a+b) /(1-r)$. Because $2 b$ of the species react among all the reactive species, the connectivity $(p)$ can be expressed as follows:

$$
p=\frac{2 b(1-r)}{a+b}
$$

Figure 4 shows a plot of $p$ as a function of $r$. p proportionally increased with an increase in $r$ in each graph. Notably, the values of $p$ are the equilibrium values because the activated esters dissociated and lose their reactivity. For $\phi_{0}=0.034$, the slope of the linear fit was $\sim 2.0$. When all the activated ester groups react with the excess amino groups, the slope was 2 . This result indicates that approximately all tetra-PEG-OSu that exist in the solution react with excess tetra-PEG-NH $\mathrm{N}_{2}$. This reaction conversion is comparable to that of the stoichiometric system, ${ }^{21,28,29,32}$ which suggests that the reaction proceeds efficiently even under stoichiometrically unbalanced conditions. The slope slightly decreased with a decrease in $\phi_{0}$, suggesting a decrease in the reaction conversion under dilute conditions. The values of $p^{*}$ were determined as $p$ at $r_{\mathrm{c}}$ for each $\phi_{0}$.

\section{Comparison with the site-bond percolation model}

Figure 5 shows the concentration-dependence of $p^{*}$. Here, the polymer volume fraction $\left(\phi_{0}\right)$ is normalized by the overlapping volume fraction $\left(\phi^{*}=0.034\right)$ predetermined by rheometry. ${ }^{29}$ The value of $p^{\star}$ decreased with an increase in $\phi_{0} / \phi^{*}$, suggesting that higher connectivity is needed to form a 3D network in the region of lower $\phi_{0} / \phi^{*}$. In our system, the lowest concentration at which a gel was formed (critical concentration) was $\sim \phi^{\star} / 6$. This result is different from the expectation from previous studies, which identified the critical concentration as the overlapping concentration. ${ }^{33,34}$ Our results indicate that gelation occurred far below the overlapping concentration of the polymers, and the non-gelation that was observed in previous studies may be caused by experimental problems that inhibit gelation, such as low-reaction efficiency and progressive intramolecular reactions. The overlapping of the polymers at the starting condition may not be so important because $\phi^{*}$, which is determined by the molecular weight of the polymer in the system, decreases as the reaction progresses. When the reaction proceeds, the polymers are connected to each other, and the average molecular weight of polymers increases. Because an increase in the molecular weight of a polymer $(M)$ decreases the overlapping volume faction as $\phi^{*} \sim M^{-0.80}$ (for real linear polymer), ${ }^{35} \phi_{0} / \phi^{*}$ increased as the reaction progressed and finally became $>1$, at which point the system was filled with polymers.

To further discuss the relationships between $p^{\star}$ and $\phi_{0} / \phi^{*}$, we use the site-bond percolation model. ${ }^{20}$ This model has two independent parameters (that is, the occupied site fraction $\left(p_{\mathrm{s}}\right)$ and the connected bond fraction between the neighboring sites $\left.\left(p_{\mathrm{b}}\right)\right)$. For simple bond percolation and site percolation, the exact values of $p_{\mathrm{s}}$ and $p_{\mathrm{b}}$ have been estimated for various lattices. However, for site-bond percolation, the exact solution is not available. Based on Monte Carlo simulations for site-bond percolation on several two-dimensional and 3D lattices ${ }^{19,36}$ as well as renormalization group studies, ${ }^{37}$ the following empirical relationship has been proposed.

$$
\frac{\log p_{\mathrm{s}}}{\log p_{\mathrm{s}}^{*}}+\frac{\log p_{\mathrm{b}}}{\log p_{\mathrm{b}}^{*}}=1
$$

where $p_{\mathrm{s}}^{*}$ and $p_{\mathrm{b}}^{*}$ are the critical fractions in the pure bond and site problems, respectively. Equation (2) indicates that the critical point of the site-bond percolation model is determined by the distance from the critical points of the site and bond percolations. For a 3D diamond lattice, $p_{\mathrm{s}}^{*}=0.43$ and $p_{\mathrm{b}}^{*}=0.39 .{ }^{38}$ Because these critical values barely change with the lattice structure, we do not lose any generality with this assumption. Here, it is important to note that we cannot directly compare $p_{\mathrm{b}}$ and the experimentally estimated reaction conversion $(p)$. The value of $p$ is estimated as the ratio of the reacted arms to all the arms. However, $p_{\mathrm{b}}$ is the probability to form a bond with a neighboring site 'only if the neighboring site is occupied'. The experimentally estimated $p$ corresponds to $p_{\mathrm{b}} p_{\mathrm{s}}$. The relationship between the theoretical prediction of $p^{*}\left(=p_{\mathrm{b}} p_{\mathrm{s}}\right)$ and $p_{\mathrm{s}}$ is shown in Figure 5 as a solid line. One of the features of the model prediction is a discontinuous change in $p^{*}$ at $p_{\mathrm{s}}=0.43$. At $p_{\mathrm{s}}=0.43$, all the neighboring sites are bonded $\left(p_{\mathrm{b}}=1\right.$ and $\left.p^{*}=0.43\right)$, and the system is just percolating. Below this point, no gel was formed even when all the neighboring sites are connected. However, in the $p_{\mathrm{s}}>0.43$ region, $p^{*}$ slightly decreased with an increase in $p_{s}$ and reached the value of the bond-percolation model $(=0.39)$ at $p_{s}=1$.

Based on the definition of $\phi^{*}$, it may be natural to find a similarity between $p_{\mathrm{s}}$ and $\phi_{0} / \phi^{*}$ because both conditions units fill the space. Here, we accept the assumption that $\phi_{0} / \phi^{\star}$ corresponds to $p_{\mathrm{s}}$ and compare the experimental data and the model prediction. When we focus on the $\phi_{0} / \phi^{*} \approx 1$ region, the experimentally estimated $p^{\star}(=0.36)$ is close to $p_{\mathrm{b}}{ }^{*}=0.39$, which suggests the applicability of the bond-percolation model to the system around $\phi^{\star}$. However, a qualitative discrepancy exists in the vicinity of the site-percolation threshold $\left(\phi_{0} / \phi^{*}=p_{s}^{*}\right): p^{*}$ shows a continuous change as a function of $\phi_{0} / \phi^{*} \cdot p^{\star}$ predicted by the site-bond percolation model shows a discontinuous change as a function of $p_{\mathrm{s}}$. This discrepancy suggests the 
a

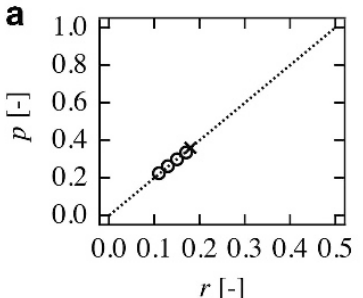

C

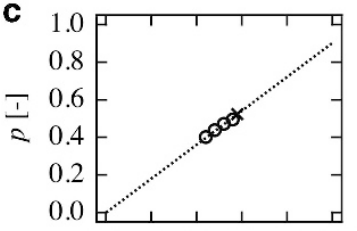

$\begin{array}{llllllllll}0 & 0.1 & 0.2 & 0.3 & 0.4 & 0.5\end{array}$

$r[-]$

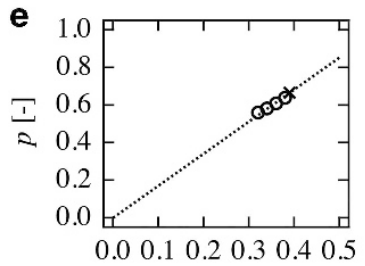

$r[-]$

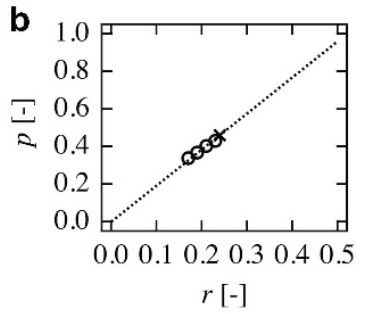

d

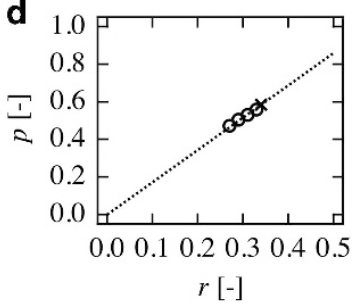

f

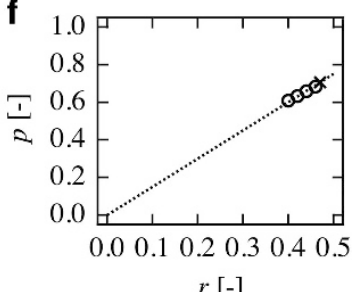

$r[-]$

Figure 4 The reaction conversion $(p)$ as a function of $r$ in (a) $\phi_{0}=0.034$, (b) $\phi_{0}=0.017$, (c) $\phi_{0}=0.011$, (d) $\phi_{0}=0.0087$, (e) $\phi_{0}=0.0070$ and (f) $\phi_{0}=0.0061$. The dashed line is the fitted curve (circle, experimental conversion; cross, critical conversion estimated by the fitting curve).

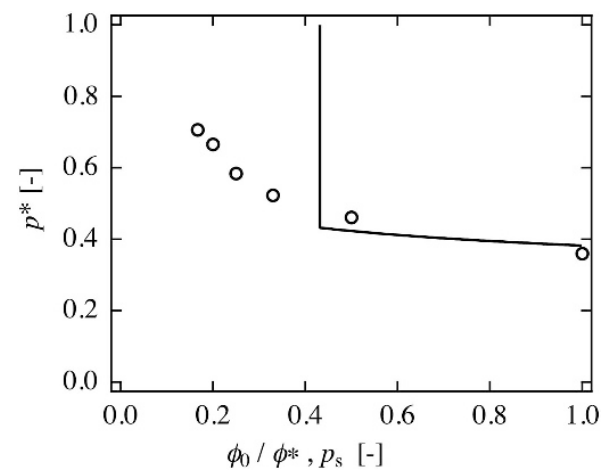

Figure 5 The critical reaction conversion $\left(p^{*}\right)$ as a function of the polymer volume fraction at a preparation state normalized by the overlapping fraction $\left(\phi_{0} / \phi^{*}\right)$. The solid line represents the prediction from the site-bond percolation model.

inapplicability of the site-percolation model to the sol-gel transition far below $\phi^{\star}$, which may originate from the limitation of the lattice model.

\section{Viscoelasticity at the critical point}

In the previous section, we discussed the phase diagram of the relationships between the critical reaction conversion $\left(p^{*}\right)$ and the polymer volume fraction at the preparation state $\left(\phi_{0}\right)$. The site-bond percolation model failed to describe the phase diagram, especially in the low $\phi_{0}$ region. To investigate the reason for this failure, we

investigated the network structure at $p^{\star}$ using rheometry. Figure 6 shows the $\omega$ dependence of $G^{\prime}$ and $G^{\prime \prime}$ at the critical point at various preparation concentrations $\left(\phi_{0}=0.0087-0.034\right)$. Under each critical condition, a power law relationship $\left(G^{\prime} \approx G^{\prime \prime} \sim \omega^{u}\right)$ was observed. The power $(u)$ relates to the percolated structure. We estimated the values of $u$ from the slope and plotted against $\phi_{0 /} \phi^{*}$ in Figure 7 . The value of $u$ decreased from 0.83 to 0.69 with an increase in $\phi_{0}$. Similar tendencies have been observed in previous studies. Winter and Chambon found that stoichiometrically unbalanced end-linked elastomers exhibited higher exponent values $(0.5<u<1),{ }^{13}$ and the stoichiometrically balanced end-linked elastomers (polydimethylsiloxane, ${ }^{17}$ polyurethane ${ }^{39}$ ) exhibited a specific value of $u=0.50$. Takahashi et al. reported that the end-linked PDMS elastomers exhibited a higher exponent at the critical point when the functional groups of the precursor chains ends were less active. ${ }^{40}$ According to these results, an increase in the effective network concentration tends to decrease the power of the critical behavior.

\section{Fractal dimension at the critical point}

According to dynamics scaling theory, ${ }^{14}$ a general expression for $u$ is as follows: ${ }^{16,41}$

$$
u=\frac{z}{z+k}
$$

Here, $z$ and $k$ are the critical exponents for the equilibrium elastic modulus and zero-shear viscosity near the critical point, respectively. When the hydrodynamic interaction is completely screened out and the excluded volume effect is dominant, equation (3) is reduced to the following form: ${ }^{42-44}$

$$
u=\frac{d}{D+2}
$$

Here, $D$ and $d$ represent the fractal dimension and the spatial dimension $(d=3)$, respectively. Using equation (4), we estimated $D$ from $u$ for each critical point and plotted them as a function of $\phi_{0 /} \phi^{*}$ in Figure 8. The value of $D$ at $\phi_{0 /} \phi^{*}=1(D \approx 2.3)$ is close to the prediction from the conventional-percolation model in $3 \mathrm{D}$ space for the diamond lattice $(D=2.5) \cdot{ }^{17}$ When the values of $\phi_{0 /} \phi^{*}$ decreased, $D$ deviates downward from the prediction of the percolation model. Notably, the site-bond percolation model always predicts $D=2.5$ for the $3 \mathrm{D}$ diamond lattice. The $3 \mathrm{D}$ diamond lattice has only a family of percolated structures with $D=2.5$ regardless of $p_{s}$. This deviation also indicates the inapplicability of the lattice-based percolation model to the concentration region below $\phi^{*}$. To understand the sol-gel transition in the dilute region, we need to remove the restriction of lattices.

Several off-lattice models have been proposed to account for the deviation of the network forming process from the percolation model. The aggregation model is a popular model for considering the network forming process. ${ }^{45,46}$ The two subtypes of aggregation processes include monomer-cluster and cluster-cluster aggregation models. The monomer-cluster aggregation model considers the growing process in which monomer particles freely diffuse and adsorb onto a spatially immobilized cluster. ${ }^{47}$ On the other hand, the cluster-cluster aggregation model considers a growing process in which the clusters and monomers diffuse and are connected to each other. The growth process is dominated by the association between the clusters. ${ }^{45,46}$ These models seem appropriate for dilute systems because the effect of diffusion is expected to become important especially in the dilute region, where clusters cannot find the reactive neighbors without diffusion. On the basis of 3D Monte Carlo simulations, the 


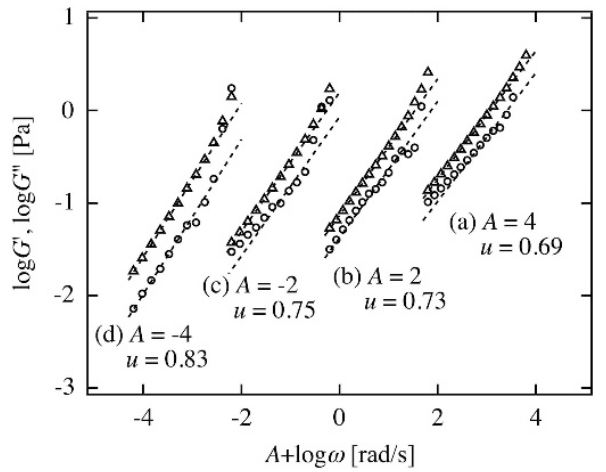

Figure 6 The values of $G^{\prime}$ and $G^{\prime \prime}$ as a function of $\omega$ for samples prepared at (a) $\phi_{0}=0.034$, (b) $\phi_{0}=0.017$, (c) $\phi_{0}=0.011$ and (d) $\phi_{0}=0.0087$. The dashed lines are the fitted curves showing the following relationship: $G^{\prime} \approx G^{\prime \prime} \sim \omega^{u}$. The curves were shifted sideways (factor $A$ ) to avoid overlap.

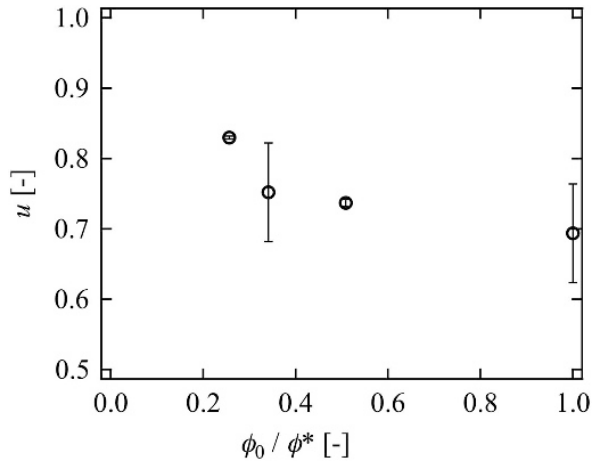

Figure 7 The $\phi_{0} / \phi^{*}$ dependence of $u$.

fractal dimensions of the percolation cluster have been estimated for the monomer-cluster (3.00 for reaction limited, and 2.50 for diffusion limited) and cluster-cluster (2.10 for reaction limited, and 1.78 for diffusion limited) aggregation processes. ${ }^{46,48}$ Our experimental value at $\phi_{0}=\phi / 4^{*}(D=1.6)$ is close to that predicted by the diffusion-limited cluster-cluster aggregation process $(D=1.76)$. This agreement suggests that the network formation process shifts from the bond-percolation model to a diffusion-limited cluster-cluster aggregation process with a decrease in $\phi_{0}$. To further examine this hypothesis, the size distribution of the clusters during the gelation should be studied. However, this investigation is beyond the scope of this paper.

Finally, we make a conjecture on the percolated network structure formed at $\phi_{0}=\phi / 4^{*}$. Despite a low $\phi_{0}$, the reaction conversion was as high as 0.8 , indicating that a large portion of the polymers can find reactive neighbors. To achieve such a high conversion with limited reactive neighbors, intramolecular reactions must occur between neighboring polymers. This prediction is supported by the abnormally low-fractal dimension $(D=1.6)$, which is close to that of the real chain $(D=1.7)$. When intramolecular reactions occur between neighboring polymers, multi-cyclic structures are most likely formed (Figure 9). This structure is locally oriented and may have a fractal dimension that is similar to that of a real chain. This locally oriented structure efficiently expands the network structure and enables the system to percolate with a limited number of polymers. The existence of this cyclic structure has been strongly suggested in our previous studies as well. ${ }^{21,22,28,29,32,49,50}$

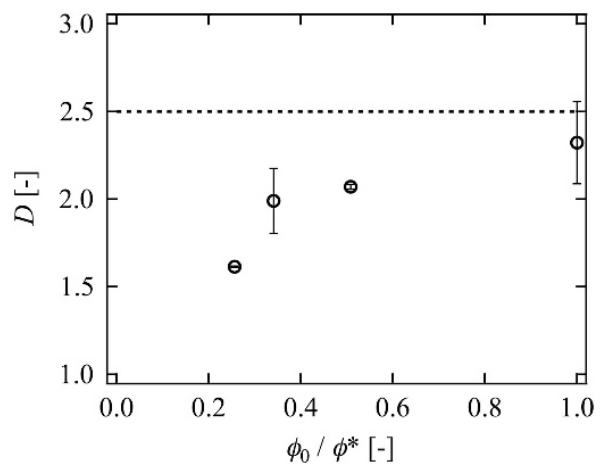

Figure 8 The $\phi_{0} / \phi^{*}$ dependence of $D$. The dashed line represents the prediction of the percolation theory.

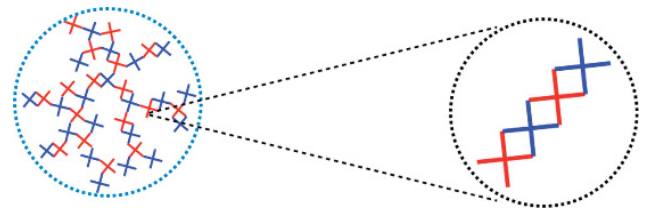

Figure 9 Schematic illustration of the chain-like structure formed at a lowpreparation concentration. The right inset shows the chain-like structure formed by intramolecular reactions.

\section{CONCLUSION}

We investigated the sol-gel phase diagram by independently tuning the connectivity and concentration using the tetra-PEG gel system with unbalanced stoichiometry. We obtained the following results: (1) the constructed phase diagram indicates that the reaction conversion required to percolate a system continuously increased with a decrease in the polymer concentration; and (2) the fractal dimension of the percolation clusters deviated from the prediction of the percolation model with a decrease in the concentration. These results validate the lattice-based percolation model around the overlapping concentration and indicate a failure of this model far below the overlapping concentration. In the region far below the overlapping concentration, diffusion of the polymers becomes important, and a multi-cyclic structure may be formed. These data will aid in elucidating the gelation process of polymer gels.

\section{CONFLICT OF INTEREST}

The authors declare no conflict of interest.

\section{ACKNOWLEDGEMENTS}

This work was supported by the Japan Society for the Promotion of Science (JSPS) through the Grants-in-Aid for the Graduate Program for Leaders in Life Innovation (GPLLI); the International Core Research Center for Nanobio; Core-to-Core Program A, Advanced Research Networks; and the Grants-in-Aid for JSPS Fellows Grant Number 12J07980 to TK, for Young Scientists (A) Grant Number 23700555 to TS, and Scientific Research (A) Grant Number 24240069 to UC. This work was also supported by the Japan Science and Technology Agency (JST) through the S-innovation program (to UC) and PREST (to TS).

\footnotetext{
1 Adam, M. Growth-process of polymers near the gelation threshold. Makromol. Chem. Macromol. Symp. 45, 1-9 (1991).

2 Fang, L., Brown, W. \& Konak, C. Dynamic light scattering study of the sol-gel transition.
} Macromolecules 24, 6839-6842 (1991). 
3 Lang, P. \& Burchard, W. Dynamic light scattering at the gel point. Macromolecules 24, 814-815 (1991).

4 Martin, J. E., Wilcoxon, J. \& Adolf, D. Critical exponents for the sol-gel transition. Phys. Rev. A 36, 1803-1810 (1987).

5 Martin, J. E., Wilcoxon, J. \& Odinek, J. Decay of density fluctuations in gels. Phys. Rev. A 43, 858-872 (1991).

6 Martin, J. E. \& Wilcoxon, J. P. Critical dynamics of the sol-gel transition. Phys. Rev. Lett. 61, 373-376 (1988).

7 Ren, S. Z., Shi, W. F., Zhang, W. B. \& Sorensen, C. M. Anomalous diffusion in aqueous solutions of gelatin. Phys. Rev. A 45, 2416-2422 (1992).

8 Adam, M., Delsanti, M. \& Durand, D. Mechanical measurements in the reaction bath during the polycondensation reaction, near the gelation threshold. Macromolecules 18, 2285-2290 (1985).

9 Adam, M., Delsanti, M., Durand, D., Hild, G. \& Munch, J. P. Mechanical-properties near gelation threshold, comparison with classical and $3 \mathrm{~d}$ percolation theories. Pure Appl. Chem. 53, 1489-1494 (1981).

10 Takigawa, T., Kasihara, H., Urayama, K. \& Masuda, T. Critical-behavior of modulus of poly(vinylalcohol) gels near the gelation point. J. Phys. Soc. Jpn. 59, 2598-2599 (1990).

11 Takigawa, T., Urayama, K. \& Masuda, T. Critical-behavior of the intrinsic-viscosity of poly (vinylalcohol) solutions near the gelation point. J. Chem. Phys. 93, 7310-7313 (1990).

12 Takigawa, T., Urayama, K. \& Masuda, T. Critical-behavior of the specific viscosity of poly(vinyl alcohol) solutions near the gelation threshold. Chem. Phys. Lett. 174, 259-262 (1990).

13 Chambon, F. \& Winter, H. H. Linear viscoelasticity at the gel point of a crosslinking PDMS with imbalanced stoichiometry. J. Rheol. 31, 683-697 (1987).

14 Martin, J. E., Adolf, D. \& Wilcoxon, J. P. Viscoelasticity near the sol-gel transition. Phys. Rev. A 39, 1325-1332 (1989).

15 Winter, H. \& Mours, M. Advances in Polymer Science, Vol. 134, Ch. 3, 165-234 (Springer, Berlin Heidelberg, 1997).

16 Winter, H. H. in Progress in Colloid \& Polymer Science Vol. 75, Ch. 13, 104-110 (Springer, Berlin Heidelberg, 1987).

17 Winter, H. H. \& Chambon, F. Analysis of linear viscoelasticity of a crosslinking polymer at the gel point. J. Rheol. 30, 367-382 (1986).

18 Stauffer, D. \& Aharony, A. Introduction To Percolation Theory, 2nd edn (Taylor \& Francis, London, UK, 1994).

19 Stauffer, D., Coniglio, A. \& Adam, M. Gelation and critical phenomena. Adv. Polym. Sci. 44, 103-158 (1982).

20 Coniglio, A., Stanley, H. E. \& Klein, W. Site-bond correlated-percolation problem: a statistical mechanical model of polymer gelation. Phys. Rev. Lett. 42, 518-522 (1979).

21 Sakai, T. Gelation mechanism and mechanical properties of Tetra-PEG gel. React. Funct. Polym. 73, 898-903 (2013).

22 Sakai, T. Experimental verification of homogeneity in polymer gels. Polym. J. 46, 517-523 (2014)

23 Sakai, T., Akagi, Y., Matsunaga, T., Kurakazu, M., Chung, U. I. \& Shibayama, M. Highly elastic and deformable hydrogel formed from tetra-arm polymers. Macromol. Rapid Commun. 31, 1954-1959 (2010).

24 Kondo, S., Chung, U.-I. \& Sakai, T. Effect of prepolymer architecture on the network structure formed by AB-type crosslink-coupling. Polym. J. 46, 14-20 (2013).

25 Sakai, T., Matsunaga, T., Yamamoto, Y., Ito, C., Yoshida, R., Suzuki, S., Sasaki, N., Shibayama, M. \& Chung, U. I. Design and fabrication of a high-strength hydrogel with ideally homogeneous network structure from tetrahedron-like macromonomers. Macromolecules 41, 5379-5384 (2008).

26 Nishi, K., Fujii, K., Chijiishi, M., Katsumoto, Y., Chung, U. I., Sakai, T. \& Shibayama, M. Kinetic study for AB-type coupling reaction of tetra-arm polymers. Macromolecules 45, 1031-1036 (2012).
27 Nishi, K., Fujii, K., Katsumoto, Y., Sakai, T. \& Shibayama, M. Kinetic aspect on gelation mechanism of tetra-PEG hydrogel. Macromolecules 47, 3274-3281 (2014).

28 Akagi, Y., Katashima, T., Katsumoto, Y., Fujii, K., Matsunaga, T., Chung, U. I., Shibayama, M. \& Sakai, T. Examination of the theories of rubber elasticity using an ideal polymer network. Macromolecules 44, 5817-5821 (2011).

29 Akagi, Y., Gong, J. P., Chung, U.-I. \& Sakai, T. Transition between phantom and affine network model observed in polymer gels with controlled network structure. Macromolecules 46, 1035-1040 (2013).

30 Matsunaga, T., Sakai, T., Akagi, Y., Chung, U. \& Shibayama, M. Structure characterization of tetra-PEG gel by small-angle neutron scattering. Macromolecules $\mathbf{4 2}$, 1344-1351 (2009)

31 Ferry, J. D. Viscoelastic Properties of Polymers, 3rd edn (Wiley, New York, Chichester, Brisbane, Tronto and Singapore, 1980).

32 Sakai, T., Kurakazu, M., Akagi, Y., Shibayama, M. \& Chung, U. Effect of swelling and deswelling on the elasticity of polymer networks in the dilute to semi-dilute region. Soft Matter 8, 2730-2736 (2012).

33 Takeda, M., Norisuye, T. \& Shibayama, M. Critical dynamics of cross-linked polymer chains near the gelation threshold. Macromolecules 33, 2909-2915 (2000).

34 Miura, T., Okumoto, H. \& Ichijo, H. Concentration dependence of the sol-gel transition point and the network formation of polymer gels. Phys. Rev. E 54, 6596-6602 (1996)

35 de Gennes, P. G. Scaling Concepts In Polymer Physics, 1st edn (Cornell University Press, Ithaca and London, 1979).

36 Yanuka, M. \& Englman, R. Bond-site percolation: empirical representation of critical probabilities. J. Phys. A Gen. Phys. 23, L339 (1990).

37 Nakanishi, H. \& Reynolds, P. J. Site-bond percolation by position-space renormalization group. Phys. Lett. A 71, 252-254 (1979).

$38 \mathrm{Xu}, \mathrm{X}$., Wang, J., Lv, J.-P. \& Deng, Y. Simultaneous analysis of three-dimensional percolation models. Front. Phys. 9, 113-119 (2014).

39 Chambon, F., Petrovic, Z. S., MacKnight, W. J. \& Winter, H. H. Rheology of model polyurethanes at the gel point. Macromolecules 19, 2146-2149 (1986).

40 Takahashi, M., Yokoyama, K., Masuda, T. \& Takigawa, T. Dynamic viscoelasticity and critical exponents in sol-gel transition of an end-linking polymer. J. Chem. Phys. 101, 798-804 (1994).

41 Hess, W., Vilgis, T. A. \& Winter, H. H. Dynamical critical behavior during chemical gelation and vulcanization. Macromolecules 21, 2536-2542 (1988).

42 Muthukumar, M. Dynamics of polymeric fractals. J. Chem. Phys. 83, 3161-3168 (1985).

43 Muthukumar, M. Screening effect on viscoelasticity near the gel point. Macromolecules 22, 4656-4658 (1989)

44 Nicolai, T., Randrianantoandro, H., Prochazka, F. \& Durand, D. Viscoelastic relaxation of polyurethane at different stages of the gel formation. 2. sol-gel transition dynamics. Macromolecules 30, 5897-5904 (1997).

45 Kolb, M., Botet, R. \& Jullien, R. Scaling of kinetically growing clusters. Phys. Rev. Lett. 51, 1123-1126 (1983)

46 Meakin, P. Diffusion-controlled cluster formation in 2-6-dimensional space. Phys. Rev. A 27, 1495-1507 (1983).

47 Witten, T. A. \& Sander, L. M. Diffusion-limited aggregation, a kinetic critical phenomenon. Phys. Rev. Lett. 47, 1400-1403 (1981).

48 Eden, M. in Proceedings of the Fourth Berkeley Symposium on Mathematical Statistics and Probability, Contributions to Biology and Problems of Medicine, vol. 4, 223-239 (University of California Press, Berkeley, CA, USA, 1961).

49 Akagi, Y., Matsunaga, T., Shibayama, M., Chung, U. \& Sakai, T. Evaluation of topological defects in tetra-PEG gels. Macromolecules 43, 488-493 (2010).

50 Katashima, T., Kurakazu, M., Akagi, Y., Chung, U.-I. \& Sakai, T. Relationships between mechanical properties of polymer gels and polymer volume fractions at preparation and at interested state. Nihon Reoroji Gakk. 42, 97-102 (2014). 